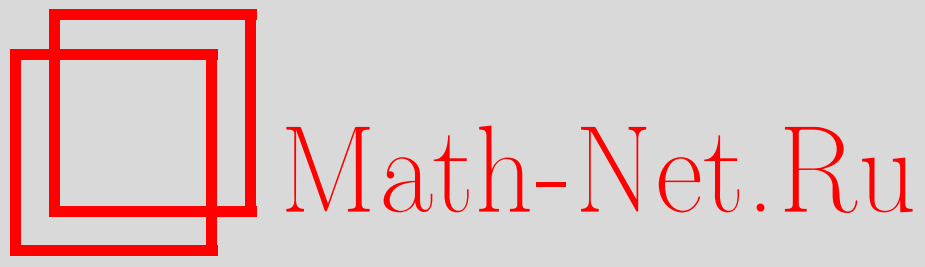

Ф. Г. Маликов, И. Б. Френкель, Аннулирующие идеалы и наклонные функторы, Функи. анализ и его прил., 1999, том 33, выпуск 2, 31-42

DOI: https://doi.org/10.4213/faa352

Использование Общероссийского математического портала MathNet.Ru подразумевает, что вы прочитали и согласны с пользовательским соглашением http://www.mathnet.ru/rus/agreement

Параметры загрузки:

IP : 35.174 .16 .151

26 апреля 2023 г., 09:53:00

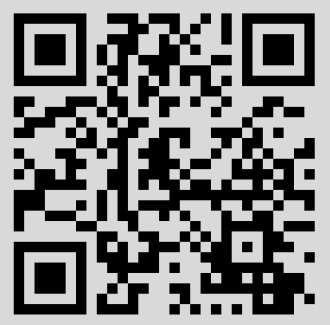


Функииональньй анализ и его приложения

1999, т. 33, вып. 2, с. 31-42

УДК 512.554 .3

\title{
Аннулирующие идеалы и наклонные функторы
}

\author{
(C) 1999. Ф. Г. МАлИКов, И. Б. ФРЕНКЕЛЬ
}

\section{$\S 1$. Введение}

Сформулируем два основных результата настоящей статьи.

(А) Пусть $\omega$ - отображение, которое подмодуль модуля Вейля над аффинной алгеброй Ли переводит в аннулирующий идеал соответствующего фактормодуля. Тогда $\omega$ устанавливает эквивалентность решетки подмодулей модуля Вейля с регулярным доминантным старшим весом и решетки вертексных идеалов локального пополнения соответствующей универсальной обертывающей алгебры. Заметим, что слова «регулярный доминантный» означают, в частности, что уровень $k$ является положительным рациональным числом; cp. $\S 2$.

(В) Пусть теперь уровень $k$ - отрицательное рациональное число, а $\mathscr{O}_{k}^{\lambda}$ категория модулей над аффинной алгеброй Ли на уровне $k$, удовлетворяющих следуюшему условию: если $A \in \mathscr{O}_{k}^{\lambda}$, то старший вес любого неприводимого подфактора модуля $A$ принадлежит орбите веса $\lambda$ под действием аффинной группы Вейля; мы предполагаем, что $\lambda$ - регулярный антидоминантный вес. Пусть далее ${ }^{\text {fam } \mathscr{O}_{k}^{\lambda}} \subset \mathscr{O}_{k}^{\lambda}$ - полная подкатегория, состоящая из модулей, «аналитически зависящих от $k$ ». Тогда категория наклонных функторов, отобра-

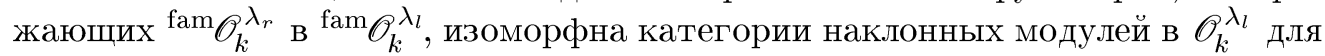
любых $\lambda_{r}, \lambda_{l}$. Если к тому же $\lambda_{r}=\lambda_{l}$, то категория наклонных функторов является моноидальной и ее кольцо Гротендика изоморфно групповой алгебре аффинной группы Вейля.

Настоящая статья является результатом попытки перенести классическую теорию бимодулей Хариш-Чандры на случай аффинной алгебры Ли. Читатель, знакомый с классической теорией, заметит, что результат (А) аналогичен известной теореме Джозефа [18] о решетке двусторонних идеалов универсальной обертывающей алгебры простой алгебры Ли, а (В) параллелен классификации проективных функторов, полученной Бернштейном и Гельфандом в [3]. Полезно также обратить внимание на существенные различия: в первом случае место ассоциативной алгебры занимает алгебра Ли, соответствующая вертексной алгебре; во втором вместо проективных функторов рассматриваются наклонные функторы - это основное новое понятие, введенное в настоящей статье. Нашим главным техническим средством является бифунктор Каждана-Люстига; в аффинном случае он играет роль функтора тензорного умножения на конечномерный модуль, успешно использованного в [3] для получения классификации бимодулей Хариш-Чандры в классическом случае.

Пользуемся случаем, чтобы поблагодарить П. Этингофа, Б. Фейгина и Г. Цуккермана за полезные обсуждения и И. Мирковича, который сообщил 
нам о своей неопубликованной работе [25], содержащей результаты, напоминающие некоторые из наших. Часть этой работы была выполнена во время посещения авторами Института Шрёдингера в Вене в 1996 г.

\section{$\S 2$. Предварительные сведения}

2.1. Ниже приводится список основных понятий и обозначений, которыми мы будем пользоваться без дальнейших пояснений:

- простая конечномерная алгебра Ли g с фиксированным треугольным разложением, в частности, с фиксированной подалгеброй Картана $\mathfrak{h} \subset \mathfrak{g} ;$ по умолчанию под двойственным пространством $\mathfrak{h}^{*}$ понимается действительная часть $\mathfrak{h}_{\mathbb{R}}^{*} ;$

- действие $(\lambda \mapsto w \lambda)$ и сдвинутое с помощью $\rho$ действие $(\lambda \mapsto w \cdot \lambda)$ группы Вейля $W$ на $\mathfrak{h}^{*}$, сохраняющее решетку весов $P \subset \mathfrak{h}^{*} ;$ обозначим через $\bar{C}$ камеру Вейля - ту фундаментальную область для сдвинутого на $\rho$ действия группы $W$, которая определяется зафиксированным треугольным разложением; $P^{+}=P \cap C$, где $C \subset \bar{C}$ есть внутренность;

- категория $\mathscr{O}_{\mathfrak{g}} \mathfrak{g}$-модулей, связанная с треугольным разложением;

- модуль Верма $M_{\lambda} \in \mathscr{O}_{\mathfrak{g}}, \lambda \in \mathfrak{h}^{*}$, и простой конечномерный модуль $V_{\lambda}, \lambda \in$ $P^{+} \subset P$

- аффинная алгебра Ли $\hat{\mathfrak{g}}=\mathfrak{g} \otimes \mathbb{C}((z)) \oplus \mathbb{C} K$ и «обобщенная» подалгебра Бореля $\hat{\mathfrak{g}}_{\geqslant}=\mathfrak{g} \otimes \mathbb{C}[[z]] \oplus \mathbb{C} K$;

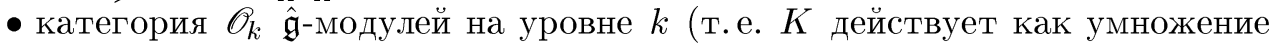
на $k$ ) и полная подкатегория $\widetilde{\mathscr{O}}_{k} \subset \mathscr{O}_{k}$, состоящая из модулей, полупростых относительно $\mathfrak{g} \hookrightarrow \hat{\mathfrak{g}}$;

- модуль Верма $M_{\lambda}^{k}=\operatorname{Ind}_{\hat{\mathfrak{g}} \geqslant}^{\hat{\mathfrak{g}}} M_{\lambda} \in \mathscr{O}_{k}, \lambda \in \mathfrak{h}^{*} ;$ модуль Вейля $V_{\lambda}^{k}=\operatorname{Ind}_{\hat{\mathfrak{g}} \geqslant}^{\hat{\mathfrak{g}}} V_{\lambda} \in$ $\widetilde{\mathscr{O}}_{k}, \lambda \in P^{+}$; более общим образом, если $V \in \mathscr{O}_{\mathfrak{g}}-$ некоторый $\mathfrak{g}$-модуль, то $V^{k} \in \mathscr{O}_{k}$ - это $\hat{\mathfrak{g}}$-модуль, полученный индуцированием с $V$; очевидно, что $V_{\lambda}^{k}$ является фактором модуля $M_{\lambda}^{k}$; каждый простой модуль из $\mathscr{O}_{k}$ - это фактор модуля $M_{\lambda}^{k}$ для некоторого $\lambda$; обозначим этот модуль через $L_{\lambda}^{k}$;

- если $k \notin \mathbb{Q}$, то $\widetilde{\mathscr{O}}_{k}$ полупроста, причем любой объект является прямой суммой модулей Вейля; эквивалентность $\mathscr{O}_{k} \approx \mathscr{O}_{\mathfrak{g}}$, доказанная Финкельбергом [11], есть аналог этого утверждения для $\mathscr{O}_{k}$;

- для $k+h^{\vee}=p / q \in \mathbb{Q}>$ рассмотрим аффинную группу Вейля $W_{k}=$ $p Q \propto W$, где $Q$ - решетка корней алгебры Ли $\mathfrak{g} ;$ имеется обычное и сдвинутое на аффинный вес $\rho$ действие группы $W_{k}$ на $\mathfrak{h}^{*} ;$ для последнего фундаментальной областью является $\bar{C}_{\mathrm{aff}}=\bar{C} \cap\{\lambda: 0 \leqslant(\lambda+\rho, \theta) \leqslant \rho\}$, где $\theta-$ старший корень алгебры Ли $\mathfrak{g}$; положим $P_{k}^{+}=P^{+} \cap C_{\text {aff }}$, где $C_{\text {aff }} \subset \bar{C}_{\text {aff }}$ - внутренность; иногда бывает важно, чтобы множество $P_{k}^{+}$содержало хотя бы один ненулевой вес; для этого, в случае неклассических систем корней, $k$ должно быть достаточно велико (см., например, $[11, \S 2.6])$, что и будет предполагаться; назовем вес $\lambda \in P_{k}^{+}$доминантным (иногда доминантным весом называют пару $(\lambda, k)$, где $\lambda$ удовлетворяет этому условию); если $k+h^{\vee}=p / q \in \mathbb{Q}_{<}$, то можно определить $W_{k}$ и антидоминантный вес сходным образом;

- $\mathscr{O}_{k}=\bigoplus_{\lambda \in P_{k}^{+}} \mathscr{O}_{k}^{\lambda}$, где $\mathscr{O}_{k}^{\lambda}$ - полная подкатегория, состоящая из модулей, композиционные ряды которых содержат только $L_{w \cdot \lambda}^{k}, w \in W_{k}$ (см. [4, 27]); аналогичное разложение справедливо для $\widetilde{\mathscr{O}}_{k}$. 
Двойственность. Обозначим через $A^{d}$ полное двойственное пространства $A$. Если $A-$ модуль над алгеброй Ли, то $A^{d}$, очевидно, тоже. Если $A$ градуировано конечномерными подпространствами, то возникает ограниченное двойственное, обозначаемое через $D(A)$.

Объекты категории $\widetilde{\mathscr{O}}_{k}$ канонически градуированы. Обозначим через $D: \widetilde{\mathscr{O}}_{k} \rightarrow \widetilde{\mathscr{O}}_{k}, M \mapsto D(M)$, функтор, такой, что $\hat{\mathfrak{g}}$-модульная структура получается компонированием канонического действия на двойственном пространстве с антиавтоморфизмом $\hat{\mathfrak{g}} \rightarrow \hat{\mathfrak{g}}, g \otimes z^{n} \mapsto g \otimes(-z)^{-n}$. Аналогичным образом определяется двойственность $D: \mathscr{O}_{k} \rightarrow \mathscr{O}_{k}$.

Функторы ${ }^{d}, D$ точны.

Имеется инволюция ${ }^{-}: P^{+} \rightarrow P^{+}$, такая, что $V_{\lambda}^{d}=V_{\bar{\lambda}}$.

В завершение отметим следующий факт о геометрии весов; вместе с доказательством он появляется в качестве леммы 7.7 в [17].

ЛЕмма 2.1.1. Предположим, ито

(i) $(\lambda, k),(\mu, k) \in P_{k}^{+}$регулярны;

(ii) если $\bar{w} \in W$, то $\bar{w}(\lambda-\mu) \in P^{+}$;

(iii) $\nu$ - вес модуля $V_{\bar{w}(\lambda-\mu)}$, такой, что $w_{1} \cdot \lambda=w \cdot \mu+\nu$ для некоторых $w, w_{1} \in W_{k}$.

Тогда $w_{1}=w u \nu \in W(\lambda-\mu)$.

\section{§3. Тензорное умножение по Каждану-Люстигу}

Каждан и Люстиг [21-23] (вдохновленные Дринфельдом [5]) определили ковариантный бифунктор

$$
\widetilde{\mathscr{O}}_{k} \times \widetilde{\mathscr{O}}_{k} \rightarrow \widetilde{\mathscr{O}}_{k}, \quad A, B \mapsto A \dot{\otimes} B .
$$

Мы напомним его определение и основные свойства

\section{1. Определения.}

3.1.1. Основные объекты. Мы будем пользоваться следующими обозначениями:

$z-$ координата на $\mathbb{C P}^{1}$;

$L \mathfrak{g}^{P}, P \in \mathbb{C P}^{1}$, - алгебра петель, «сидящая» в $P$; иными словами, $L \mathfrak{g}^{P}=$ $\mathfrak{g} \otimes \mathbb{C}((z-P)), P \in \mathbb{C}$, и $L \mathfrak{g}^{\infty}=\mathfrak{g} \otimes \mathbb{C}\left(\left(z^{-1}\right)\right) ;$

более общим образом, если $P=\left\{P_{1}, \ldots, P_{m}\right\}$, то $L \mathfrak{g}^{P}=\bigoplus_{i=1}^{m} L \mathfrak{g}^{P_{i}}$;

$\hat{\mathfrak{g}}^{P}$ - аффинная алгебра, сидящая в точке $P$, - каноническое центральное расширение алгебры $L \mathfrak{g}^{P} ;$ разумеется, $\hat{\mathfrak{g}}^{0}=\hat{\mathfrak{g}}$;

более общим образом, если $P=\left\{P_{1}, \ldots, P_{m}\right\}$, то $\hat{\mathfrak{g}}^{P}-$ прямая сумма алгебры $\hat{\mathfrak{g}}^{P_{i}}, i=1, \ldots, m$, по модулю следующего соотношения: все канонические центральные элементы $K$, по одному в каждой алгебре, равны;

$\Gamma=\mathfrak{g} \otimes \mathbb{C}\left[z, z^{-1},(z-1)^{-1}\right] ;$ очевидно, что $\Gamma$ - алгебра Ли.

Разложения в ряд Лорана в точках $\infty, 1,0$ доставляют гомоморфизм алгебр Ли

$$
\varepsilon: \Gamma \rightarrow L \mathfrak{g}^{\{\infty, 1,0\}} .
$$

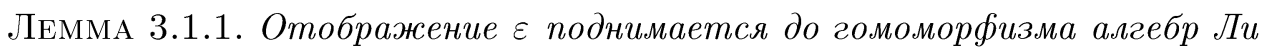

$$
\varepsilon: \Gamma \rightarrow \hat{\mathfrak{g}}^{\{\infty, 1,0\}} .
$$

Доказательство состоит в использовании теоремы о вычетах [22]. 


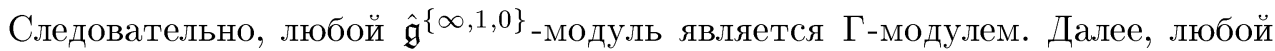
модуль $A \in \widetilde{\mathscr{O}}_{k}$ естественно наделяется структурой $\hat{\mathfrak{g}}^{P}$-модуля для любой точки $P$ с помошью очевидной замены координат. Если $A, B, C \in \widetilde{\mathscr{O}}_{k}$, рассмотрим $A \otimes B \otimes C$ как $\hat{\mathfrak{g}}^{\{\infty, 1,0\}}$-модуль, имея в виду, что $\hat{\mathfrak{g}}^{\infty}$ действует на $A, \hat{\mathfrak{g}}^{1}-$ на $B$, a $\hat{\mathfrak{g}}^{0}$ - на $C$. (В этом обозначении имеется очевидная неопределенность.) Возникает пространство коинвариантов

$$
(A \otimes B \otimes C)_{\Gamma}=(A \otimes B \otimes C) / \Gamma(A \otimes B \otimes C) .
$$

3.1.2. Определение. Пусть $\widehat{\Gamma}$ - центральное расширение алгебры Ли $Г$ с коциклом, определенным, как обычно, с той лишь разницей, что берется сумма вычетов в $\infty$ и 1 . Пусть $\Gamma(0) \subset \widehat{\Gamma}$ - подалгебра, состоящая из функций, обращающихся в нуль в 0 . Ясно, что ее можно также рассматривать как подалгебру в $\Gamma$.

Пространство $(A \otimes B)^{d}$, очевидно, является $\widehat{\Gamma}$-модулем. Он обладает возрастающей фильтрацией $\left\{(A \otimes B)^{d}(N)\right\}$, где

$$
(A \otimes B)^{d}(N)=\left\{x \in(A \otimes B)^{d}: \gamma_{1} \cdots \gamma_{N} x=0, \text { если } \gamma_{i} \in \Gamma(0) \forall i\right\} .
$$

Пространство $\bigcup_{N \geqslant 1}(A \otimes B)^{d}(N)$, естественно, является $\hat{\mathfrak{g}}$-модулем. Переход от $(A \otimes B)^{d}$ к $\bigcup_{N \geqslant 1}(A \otimes B)^{d}(N)$ часто называется функтором гладких векторов.

Положим

TEOPEMA 3.1.2.

$$
A \dot{\otimes} B=D\left(\bigcup_{N \geqslant 1}(A \otimes B)^{d}(N)\right) .
$$

(i) $\operatorname{Hom}_{\hat{\mathfrak{g}}}(A \dot{\otimes} B, D(C))=\operatorname{Hom}_{\hat{\mathfrak{g}}}(C, D(A \dot{\otimes} B))=\left((A \otimes B \otimes C)_{\Gamma}\right)^{d}$.

(ii) Если у $A, B \in \widetilde{\mathscr{O}}_{k}$ есть фильтрачия Вейля, то $A \dot{\otimes} B$ обладает тем же свойством. (Под фильтрацией Вейля мы понимаем конечную фильтрацию, последовательные факторы которой являются модулями Вейля.)

(iii) Ecли $k \notin \mathbb{Q}$, mo $V_{\lambda}^{k} \dot{\otimes} V_{\mu}^{k}=\left(V_{\lambda} \otimes V_{\mu}\right)^{k}$.

(iv) Для любого $k \in \mathbb{C}$ модуль $V_{\lambda}^{k} \dot{\otimes} V_{\mu}^{k}$ обладает фильтрачией Вейля (см. (ii)), причем кратность модуля $V_{\nu}^{k}$ равна $\left(V_{\lambda} \otimes V_{\mu}: V_{\nu}\right)$.

(v) Имеется изоморфизм $A \dot{\otimes} V_{0}^{k} \rightarrow A$ для любого $A \in \widetilde{\mathscr{O}}_{k}$.

(vi) Существуют морфизмы коммутативности и ассочиативности

$$
A \dot{\otimes} B \approx B \dot{\otimes} A, \quad(A \dot{\otimes} B) \dot{\otimes} C \approx A \dot{\otimes}(B \dot{\otimes} C),
$$

наделяющие $\widetilde{\mathscr{O}}_{k}$ структурой коммутативной моноидальной категории ${ }^{1}$.

\section{$\S 4$. Аффинные функторы сдвига}

4.1. Определение. Для любого $(\lambda, k) \in P_{k}^{+}$обозначим через $\widetilde{\mathscr{O}}_{k}^{\lambda}$ полную подкатегорию категории $\widetilde{\mathscr{O}}_{k}$, состоящую из тех модулей, старшие веса композиционных факторов которых лежат в орбите $W_{k} \cdot(\lambda, k)$. Возникает проекция

$$
p_{\lambda}: \widetilde{\mathscr{O}}_{k} \rightarrow \widetilde{\mathscr{O}}_{k}^{\lambda} \text {. }
$$

\footnotetext{
${ }^{1}$ Braided monoidal category в англоязычной литературе.
} 
Для любых двух весов $(\lambda, k),(\mu, k) \in P_{k}^{+}$выберем $\bar{w} \in W$ так, что $\bar{w}(\lambda-\mu) \in$ $P^{+}$. Очевидно, что тогда $(\bar{w}(\lambda-\mu), k) \in P_{k}^{+}$.

Определим функтор сдвига следующей формулой:

$$
T_{\mu}^{\lambda}: \widetilde{\mathscr{O}}_{k}^{\mu} \rightarrow \widetilde{\mathscr{O}}_{k}^{\lambda}, \quad A \mapsto p_{\lambda}\left(V_{\bar{w}(\lambda-\mu)}^{k} \dot{\otimes} A\right)
$$

Первым ввел такие функторы, вероятно, Финкельберг [12], который, однако, рассматривал их только при $k<0$.

В качестве очевидного следствия из определения получаем

$$
T_{\lambda}^{\mu}=p_{\mu} \circ\left(\left(V_{\bar{w}(\lambda-\mu)}^{d}\right)^{k} \dot{\otimes} ?\right) .
$$

\section{2. Жесткость модулей Вейля с доминантным старшим весом.}

ЛЕмма 4.2.1. Если $(\lambda, \mu),(\mu, k)$ регулярньл $u w \in W$ таков, ито $w \cdot \mu \in P^{+}$, mo

$$
T_{\mu}^{\lambda}\left(V_{w \cdot \mu}^{k}\right)=V_{w \cdot \lambda}^{k}
$$

ДокАЗАтЕльство. Согласно теореме 3.1.2(iv), $T_{\mu}^{\lambda}\left(V_{w \cdot \mu}^{k}\right)$ обладает фильтрацией с факторами вида $V_{w_{1} \cdot \lambda}^{k}, w_{1} \in W_{k}$, причем $w_{1} \cdot \lambda=w \cdot \mu+\nu$, где $\nu$ это вес модуля $V_{\bar{w}(\lambda-\mu)}$. По лемме $2.1 w_{1}=w$. Из этого следует, что наша фильтрация состоит из единственного члена $V_{w \cdot \lambda}^{k}$.

СлЕДСТВИЕ 4.2.2. Если $(\lambda, k) \in P_{k}^{+}$регулярен, то $V_{0}^{k}$ является прямьм слагаемьлм в $V_{\lambda}^{k} \dot{\otimes} V_{\bar{\lambda}}^{k}$.

ДокАЗАтЕльство. Конечно же, $(0, k)$ доминантен и регулярен, а $p_{0}(A)$ - прямое слагаемое в $A$. Остается заметить, что $T_{\bar{\lambda}}^{0} V_{\bar{\lambda}}^{k}=p_{0}\left(V_{\lambda}^{k} \dot{\otimes} V_{\bar{\lambda}}^{k}\right)$ и воспользоваться леммой 4.2.1, чтобы получить $T_{\bar{\lambda}}^{0} V_{\bar{\lambda}}^{k}=V_{0}^{k}$.

Таким образом, возникают отображения

$$
i_{\lambda}: V_{0}^{k} \rightarrow V_{\lambda}^{k} \dot{\otimes} V_{\bar{\lambda}}^{k}, \quad e_{\lambda}: V_{\bar{\lambda}}^{k} \dot{\otimes} V_{\lambda}^{k} \rightarrow V_{0}^{k} .
$$

Замечая, что отображения между $\dot{\otimes}$-произведениями модулей Вейля однозначно определяются их ограничениями на соответствующие конечномерные $\mathfrak{g}$-модули (теорема 3.1.2(i)), мы убеждаемся в том, что при подходящем нормировании отображений $i_{\lambda}$ и $e_{\lambda}$ композиции

$$
\begin{aligned}
& V_{\lambda}^{k}=V_{0}^{k} \dot{\otimes} V_{\lambda}^{k} \stackrel{i_{\lambda} \otimes \mathrm{id}}{\longrightarrow} V_{\lambda}^{k} \dot{\otimes} V_{\bar{\lambda}}^{k} \dot{\otimes} V_{\lambda}^{k} \stackrel{\mathrm{id} \otimes e_{\lambda}}{\longrightarrow} V_{\lambda}^{k}, \\
& V_{\bar{\lambda}}^{k}=V_{\bar{\lambda}}^{k} \dot{\otimes} V_{0}^{k} \stackrel{\mathrm{id} \otimes i_{\lambda}}{\longrightarrow} V_{\bar{\lambda}}^{k} \dot{\otimes} V_{\lambda}^{k} \dot{\otimes} V_{\bar{\lambda}}^{k} \stackrel{e_{\lambda} \otimes \mathrm{id}}{\longrightarrow} V_{\bar{\lambda}}^{k}
\end{aligned}
$$

равны единице. По определению (см., например, [23, III, Appendix]) имеем

СлЕдСтвиЕ 4.2.3. Если $(\lambda, k) \in P_{k}^{+}$, mo $V_{\lambda}^{k} u V_{\bar{\lambda}}^{k}$ жестки.

Рассмотрим функтор

$$
V_{\lambda}^{k} \dot{\otimes} ?: \widetilde{\mathscr{O}}_{k} \rightarrow \widetilde{\mathscr{O}}_{k}, \quad M \mapsto V_{\lambda}^{k} \dot{\otimes} M .
$$

СЛЕДСТВИЕ 4.2.4. (i) Пусть $(\lambda, k) \in P_{k}^{+}$. Тогда

(i) функторьи $V_{\lambda}^{k} \dot{\otimes}$ ? u $V_{\bar{\lambda}}^{k} \dot{\otimes}$ ? сопряжень, m. е. имеется естественный изоморфизм

$$
\operatorname{Hom}_{\hat{\mathfrak{g}}}\left(V_{\lambda}^{k} \dot{\otimes} A, B\right)=\operatorname{Hom}_{\hat{\mathfrak{g}}}\left(A, V_{\bar{\lambda}}^{k} \dot{\otimes} B\right) ;
$$


(ii) функторь $V_{\lambda}^{k} \dot{\otimes}$ ? u $V_{\bar{\lambda}}^{k} \dot{\otimes} ?$ точны, m. е. переводят короткие точнье последовательности в точные.

Доказательство стандартно; см., например, [23, III, Appendix].

4.3. Свойства аффинных функторов сдвига. Как известно, имеется понятие формального характера $\operatorname{ch} A, A \in \widetilde{\mathscr{O}}_{k}^{\lambda}$ (см. [4]). Возникает абелева группа характеров, причем каждое из множеств $\left\{\operatorname{ch} V_{w \cdot \lambda}^{k}, w \in W_{k}\right\},\left\{\operatorname{ch} L_{w \cdot \lambda}^{k}\right.$, $\left.w \in W_{k}\right\}$ является ее топологическим базисом. Конечно же, символы $\operatorname{ch~} V_{w \cdot \lambda}^{k}$, $\operatorname{ch} L_{w \cdot \lambda}^{k}$ нужно игнорировать, если $w \cdot \lambda \notin P^{+}$. Заметим, что

$$
\operatorname{ch} A=\sum_{w \geqslant w_{o}} \bar{n}_{w} \operatorname{ch} L_{w \cdot \mu}^{k} \Longleftrightarrow \operatorname{ch} A=\sum_{w \geqslant w_{o}} n_{w} \operatorname{ch} V_{w \cdot \mu}^{k} .
$$

ПРЕДЛОЖЕНИЕ 4.3.1. Пусть $(\lambda, k) u(\mu, k)$ регулярны и доминантньл. Тогда

(i) $T_{\mu}^{\lambda}$ точен;

(ii) $T_{\mu}^{\lambda}, T_{\lambda}^{\mu}$ сопряженья;

(iii) если $\operatorname{ch} A=\sum_{w \in W_{k}} n_{w} \operatorname{ch} V_{w \cdot \mu}^{k}, m o \operatorname{ch} T_{\mu}^{\lambda} A=\sum_{w \in W_{k}} n_{w} \operatorname{ch} V_{w \cdot \lambda}^{k}$;

(iv) $T_{\mu}^{\lambda}\left(L_{w \cdot \mu}^{k}\right)=L_{w \cdot \lambda}^{k}$;

(v) более обиим образом, $T_{\mu}^{\lambda}$ устанавливает эквивалентность решеток подмодулей модулей $V_{w \cdot \mu}^{k} u V_{w \cdot \lambda}^{k}$.

ДокАзАтельство. (i) $T_{\mu}^{\lambda}$ точен, так как является композицией точных функторов $p_{\lambda}$ и $\left(V_{\bar{w}(\lambda-\mu)}^{d}\right)^{k} \dot{\otimes} ? ;$ см. следствие $4.2 .4(\mathrm{ii})$.

(ii) Из следствия 4.2.4(i) вытекает, что для любых $A \in \widetilde{\mathscr{O}}_{k}^{\mu}, B \in \widetilde{\mathscr{O}}_{k}^{\lambda}$

$$
\begin{aligned}
\operatorname{Hom}_{\hat{\mathfrak{g}}}\left(T_{\mu}^{\lambda} A, B\right) & =\operatorname{Hom}_{\hat{\mathfrak{g}}}\left(p_{\lambda}\left(V_{\bar{w}(\lambda-\mu)}^{k} \dot{\otimes} A\right), B\right) \\
& =\operatorname{Hom}_{\hat{\mathfrak{g}}}\left(V_{\bar{w}(\lambda-\mu)}^{k} \dot{\otimes} A, B\right)=\operatorname{Hom}_{\hat{\mathfrak{g}}}\left(A,\left(V_{\bar{w}(\lambda-\mu)}^{d}\right)^{k} \dot{\otimes} B\right) \\
& =\operatorname{Hom}_{\hat{\mathfrak{g}}}\left(A, p_{\mu}\left(\left(V_{\bar{w}(\lambda-\mu)}^{d}\right)^{k} \dot{\otimes} B\right)\right)=\operatorname{Hom}_{\hat{\mathfrak{g}}}\left(A, T_{\lambda}^{\mu} B\right) .
\end{aligned}
$$

(iii) легко вытекает из (i) (если воспользоваться локальными композиционными рядами; см. [4]).

(iv) Предположим, что $T_{\mu}^{\lambda}\left(L_{w_{0} \cdot \mu}^{k}\right)$ приводим. Тогда возникает точная последовательность

$$
0 \rightarrow N \rightarrow T_{\mu}^{\lambda}\left(L_{w_{0} \cdot \mu}^{k}\right) \rightarrow L_{w_{0} \cdot \lambda}^{k} \rightarrow 0 .
$$

Применяя к ней $T_{\lambda}^{\mu}$, получаем

$$
0 \rightarrow T_{\lambda}^{\mu}(N) \rightarrow T_{\lambda}^{\mu}\left(T_{\mu}^{\lambda}\left(L_{w_{0} \cdot \mu}^{k}\right)\right) \rightarrow T_{\lambda}^{\mu}\left(L_{w_{0} \cdot \lambda}^{k}\right) \rightarrow 0 .
$$

В силу (iii) и (7) имеем $\operatorname{ch}\left(T_{\lambda}^{\mu}\left(T_{\mu}^{\lambda}\left(L_{w_{0} \cdot \mu}^{k}\right)\right)\right)=\operatorname{ch} L_{w_{0} \cdot \mu}^{k}$ и $\operatorname{ch} T_{\lambda}^{\mu}(N) \neq 0$; поэтому $\operatorname{ch} T_{\lambda}^{\mu}\left(L_{w_{0} \cdot \lambda}^{k}\right)<\operatorname{ch} L_{w_{0} \cdot \mu}^{k}$. Противоречие.

(v) Здесь доказательство является очевидной версией доказательства п. (iv); детали мы опускаем.

4.4. ТЕОРЕМА 4.4.1. Функтор $T_{\mu}^{\lambda}: \widetilde{\mathscr{O}}_{k}^{\mu} \rightarrow \widetilde{\mathscr{O}}_{k}^{\lambda}$ доставляет эквивалентность категорий.

ДокаЗАтЕЛьство. Достаточно доказать, что $T_{\mu}^{\lambda} \circ T_{\lambda}^{\mu}: \widetilde{\mathscr{O}}_{k}^{\lambda} \rightarrow \widetilde{\mathscr{O}}_{k}^{\lambda}$ эквивалентен тождественному функтору. Иными словами, надо доказать, что $\mathrm{id}: A \rightarrow A$ преобразуется в изоморфизм в $\operatorname{Hom}_{\hat{\mathfrak{g}}}\left(T_{\mu}^{\lambda} \circ T_{\lambda}^{\mu}(A), A\right)$. Это нам 
уже известно, если $A$ прост (см. предложение 4.3.1(iv)). Пользуясь локальными композиционными рядами и тем фактом, что в силу следствия $4.2 .2 A \subset$ $T_{\mu}^{\lambda} \circ T_{\lambda}^{\mu}(A)$ выделяется прямым слагаемым, можно вывести нужное утверждение в общем случае.

ЗАмЕЧАниЕ 4.4.2. Тем же способом можно определить функторы сдвига на категории $\mathscr{O}_{k}$ и доказать, что категории $\mathscr{O}_{k}^{\mu}$ и $\mathscr{O}_{k}^{\nu}$ эквивалентны, если $\mu$ и $\nu$ целочисленны, регулярны и доминантны. Единственное отличие конструкции в этом случае состоит в использовании $\dot{\otimes}$-произведения модуля Вейля с объектами всей категории $\mathscr{O} \supset \widetilde{\mathscr{O}}_{k}($ см. [12]). В частности, видно, что

$$
\left(V_{\lambda}^{k} \dot{\otimes} M_{\mu}^{k}: M_{\nu}^{k}\right)=\left(V_{\lambda} \otimes M_{\mu}: M_{\nu}\right)
$$

(cp. с теоремой 3.1.2(iv)).

\section{§5. Аннулирующие идеалы модулей со старшим весом}

5.1. Поля, коинварианты и вертексные алгебры. По теореме 3.1.2(v) $A \dot{\otimes} V_{0}^{k} \approx A$ для любого $A \in \widetilde{\mathscr{O}}_{k}$. Поэтому в силу той же теоремы $3.1 .2(\mathrm{i})$ для любого $B \in \widetilde{\mathscr{O}}_{k}$ имеется естественный изоморфизм

$$
\left(\left(A \otimes V_{0}^{k} \otimes D(B)\right)_{\Gamma}\right)^{d} \approx \operatorname{Hom}_{\hat{\mathfrak{g}}}(A, B) .
$$

Напомним, что пространство $\left(\left(A \otimes V_{0}^{k} \otimes D(B)\right)_{\Gamma}\right)^{d}$ было определено с помощью алгебры Ли $Г$, а $\Gamma$, в свою очередь, определена выбором трех точек $\infty$, 1, 0; см. п. 3.1.1. В выборе точек имеется, конечно, произвол. Зафиксировав $\infty$ и 0 и модули $A$ и $D(B)$, сидящие в них, разрешим третьей точке быть переменной. Тогда мы получим семейство алгебр Ли $\Gamma_{t}, t \in \mathbb{C}^{*}$, и семейство векторных пространств (ср. с п. 3.1.1)

$$
\left\langle A, V_{0}^{k}, D(B)\right\rangle_{t}:=\left(\left(A \times V_{0}^{k} \times D(B)\right)_{\Gamma_{t}}\right)^{d}, \quad t \in \mathbb{C}^{*} .
$$

Эти пространства естественно организуются в тривиальное линейное расслоение над $\mathbb{C}^{*}$ со слоем

$$
\left\langle A, V_{0}^{k}, D(B)\right\rangle_{t}=(A \otimes D(A))_{\tilde{\mathfrak{g}}}=\operatorname{Hom}_{\hat{\mathfrak{g}}}(A, B)
$$

в силу соображений, использующих взаимность Фробениуса. Выберем сечение этого расслоения, зафиксировав $\varphi \in \operatorname{Hom}_{\mathfrak{g}}(A, B)$. Это дает трилинейный функционал, зависящий от $t \in \mathbb{C}^{*}$,

$$
\Phi_{t}^{\varphi} \in\left\langle A, V_{0}^{k}, D(B)\right\rangle_{t} \subset\left(A \otimes V_{0}^{k} \otimes D(B)\right)^{d} .
$$

Проинтерпретируем его как линейное отображение:

$$
\Phi_{t}^{\varphi}(\cdot): V_{0}^{k} \rightarrow(A \otimes D(B))^{d},
$$

или, эквивалентным образом,

$$
\widetilde{\Phi}_{t}^{\varphi}(\cdot): V_{0}^{k} \rightarrow \operatorname{Hom}_{\mathbb{C}}\left(A, D(B)^{d}\right), \quad t \in \mathbb{C}^{*}
$$

Поскольку $\operatorname{dim} B=\infty$, образ отображения $\widetilde{\Phi}_{t}^{\varphi}$ не может быть отождествлен с $\operatorname{Hom}_{\mathbb{C}}(A, B)$. Однако простые непосредственные вычисления показывают, что $\widetilde{\Phi}_{t}^{\varphi}(v) \in \operatorname{Hom}_{\mathbb{C}}(A, B)\left[\left[t, t^{-1}\right]\right]$ для любого $v \in V_{0}^{k} ;$ иными словами, $\widetilde{\Phi}_{t}^{\varphi}(v)-$ это формальная порождающая функция семейства элементов из $\operatorname{Hom}_{\mathbb{C}}(A, B)$. Чтобы привести пример, предположим, что модуль $B$ либо равен $A$, либо 
является фактором модуля $A$, a $\mathrm{id}: A \rightarrow B$ - проекция. Чтобы согласовать наши обозначения со стандартными, положим $Y(v, t):=\widetilde{\Phi}_{t}^{\mathrm{id}}(v)$. Тогда можно показать, что

$$
Y(\operatorname{vac}, t)=\mathrm{id},
$$

где vac - вектор старшего веса модуля $V_{0}^{k}$. Далее,

$$
Y\left(\left(x \otimes z^{-1}\right) \operatorname{vac}, t\right)=\sum_{i=-\infty}^{+\infty}\left(x \otimes z^{i}\right) t^{-i-1},
$$

где $x \otimes z^{i} \in \hat{\mathfrak{g}}$ отождествлен с оператором, действующим в $A$; эта конструкция воспроизводит знаменитый ток.

Из определений легко вывести

СлЕДСТВИЕ 5.1.1. $\Phi_{t}^{\mathrm{id}}(v)(x, y)=y(Y(v, t) x)$.

Напомним, что вертексная алгебра определяется как градуированное векторное пространство $V=\bigoplus_{i \in \mathbb{Z}} V[i]$ вместе с отображением

$$
Y(\cdot, t): V \rightarrow \operatorname{End}(V)\left[\left[t, t^{-1}\right]\right],
$$

удовлетворяющим ряду аксиом, в числе которых упомянем аксиомы ассоциативности и коммутативности; см., например, $[14,15]$. Сходным образом определяется модуль над вертексной алгеброй. Вертексная алгебра является модулем над собой. Назовем идеалом вертексной алгебры ее подмодуль. Напомним также, что Фурье-компоненты полей $Y(v, t), v \in V$, образуют алгебру Ли; обозначим ее через $\operatorname{Lie}(V)$. Таким образом, идеал вертексной алгебры $V$ определяет идеал алгебры Ли $\operatorname{Lie}(V)$ в смысле алгебр Ли. Не всякий идеал алгебры Ли Lie $(V)$ может быть получен таким способом. Идеалы, полученные так, будем называть вертексныли.

Из [15] (см. также [9]) вытекает, что в силу конструкций из разд. 5.1 $\left(V_{0}^{k}, Y(\cdot, t)\right)$ - вертексная алгебра, а любой $A \in \widetilde{\mathscr{O}}_{k}$ - модуль над ней. Алгебра Ли $\operatorname{Lie}\left(V_{0}^{k}\right)$ обычно обозначается через $U(\hat{\mathfrak{g}})_{\operatorname{loc}}$ и называется локальныл пополнением алгебрь $U(\hat{\mathfrak{g}})$, хоть и не является ассоциативной алгеброй. Легко

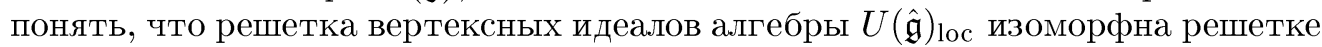
подмодулей модуля $V_{0}^{k}$, рассматриваемого как $\hat{\mathfrak{g}}$-модуль.

5.2. Здесь мы доказываем следующую теорему - один из основных результатов данной статьи.

TЕорема 5.2.1. Пусть $k \in \mathbb{Q}_{>}, a(\lambda, k),(0, k) \in P_{k}^{+}$регулярны. Обозначим через $\Omega\left(V_{\lambda}^{k}\right)$ решетку подмодулей модуля $V_{\lambda}^{k}$, а через $\Omega\left(U(\hat{\mathfrak{g}})_{\text {lос }}\right)$ решетку вертексных идеалов алгебры $U(\hat{\mathfrak{g}})_{\text {lос }}$ с иентральным зарядом $k$. Следуюшее отображение является изоморфизмом решеток:

$$
\omega: \Omega\left(U(\hat{\mathfrak{g}})_{\mathrm{loc}}\right) \rightarrow \Omega\left(V_{\lambda}^{k}\right), \quad \Omega\left(U(\hat{\mathfrak{g}})_{\mathrm{loc}}\right) \ni I \mapsto I V_{\lambda}^{k}
$$

ДоКАЗАТЕЛЬСтво. Прежде всего, по определению алгебры Ли $U(\hat{\mathfrak{g}})_{\text {loc }}$ (см. разд. 5.1) отображение $\omega$ можно эквивалентным образом рассматривать как отображение решеток подмодулей, т. е. $\omega: \Omega\left(V_{0}^{k}\right) \rightarrow \Omega\left(V_{\lambda}^{k}\right)$. Будем доказывать теорему в этой интерпретации.

Рассмотрим функтор сдвига $T_{0}^{\lambda}$. Если $N \subset V_{0}^{k}-$ подмодуль, то, с одной стороны, мы имеем

$$
T_{0}^{\lambda}\left(V_{0}^{k}\right)=V_{\lambda}^{k} \dot{\otimes} V_{0}^{k}\left(=V_{\lambda}^{k}\right)
$$


и, следовательно,

$$
T_{0}^{\lambda}\left(V_{0}^{k} / N\right)=V_{\lambda}^{k} \dot{\otimes}\left(V_{0}^{k} / N\right) .
$$

По теореме 3.1.2 и следствию 5.1.1

$$
\operatorname{Hom}_{\hat{\mathfrak{g}}}\left(T_{0}^{\lambda}\left(V_{0}^{k} / N\right), ?\right)=\left\langle V_{0}^{k} / N, V_{\lambda}^{k}, D(?)\right\rangle_{t}=\operatorname{Hom}_{\hat{\mathfrak{g}}}\left(V_{\lambda}^{k} / \omega(N), ?\right) .
$$

С другой стороны, по предложению 4.3.1(i)

$$
T_{0}^{\lambda}\left(V_{0}^{k} / N\right)=V_{\lambda} / T_{0}^{\lambda}(N) .
$$

Сравнивая последнее равенство с (13), заключаем, что $\omega(N)=T_{0}^{\lambda}(N)$. Остается вспомнить, что $T_{0}^{\lambda}$ является изоморфизмом решеток подмодулей в силу предложения 4.3.1(v).

Применим этот результат к аннулирующим идеалам допустимых представлений. Напомним, что если $k+h^{\vee} \in \mathbb{Q}_{>}$и $(\lambda, k) \in P_{k}^{+}$регулярен, то $L_{\lambda}^{k}$ называется допустимым [20]. Модуль $L_{\lambda}^{k}$ - неприводимый фактор модуля $V_{\lambda}^{k}$ по подмодулю $N_{\lambda}^{k}$, порожденному одним особым вектором; см. также [20]. По теореме 5.2.1 $\omega\left(N_{0}^{k}\right)=N_{\lambda}^{k}$. Мы доказали

СлЕдСТВИЕ 5.2.2. Аннулирующий идеал допустимого представления равен $\operatorname{Lie}\left(N_{0}^{k}\right) ;$ в частности, он порожден (в смысле вертексных алгебр) одним особилм вектором из $V_{0}^{k}$.

\section{§. Наклонные функторы - случай отрицательного центрального заряда}

Начиная с этого момента, $k+h^{\vee}$ - отрицательное рациональное число. Структура категории $\mathscr{O}_{k}$ может быть описана следующим образом. Пусть $(\lambda, k)$ антидоминантен, а $\lambda$ целочислен. Обозначим через $\mathscr{O}_{k}^{\lambda}$ полную подкатегорию, состоящую из модулей, которые в качестве подфакторов содержат только те неприводимые модули, старшие веса которых лежат в $W_{k}$-орбите веса $(\lambda, k)$. В силу $[4,27] \mathscr{O}_{k}=\bigoplus_{\lambda} \mathscr{O}_{k}^{\lambda}($ см. также $\S 2)$.

Из [19] вытекает, что любой объект из $\mathscr{O}_{k}^{\lambda}$ имеет конечную длину. Возникает кольцо Гротендика, причем каждое из множеств $\left\{\operatorname{ch}\left(M_{w \cdot \lambda}^{k}\right), w \in W_{k}\right\}$, $\left\{\operatorname{ch}\left(L_{w \cdot \lambda}^{k}\right), w \in W_{k}\right\}$ является его базисом.

Все это переносится на случай $\widetilde{\mathscr{O}}_{k}$ путем замены $M_{\lambda}^{k}$ на $V_{\lambda}^{k}$ (предварительно надо убедиться, что в этом случае $\lambda$ - целочисленный доминантный вес).

\section{1. Наклонные модули. Определение.}

Модуль $U$ из $\mathscr{O}_{k}$ (соответственно $\left.\widetilde{\mathscr{O}}_{k}\right)$ называется наклоннылм, если и $U$ и $D(U)$ обладают фильтрацией, состоящей из модулей Верма (соответственно Вейля).

Например, модуль Верма с антидоминантным старшим весом является наклонным в $\mathscr{O}_{k}$, так как он неприводим и поэтому изоморфен своему двойственному. Аналогично, модуль Вейля с антидоминантным весом тоже является наклонным в $\widetilde{\mathscr{O}}_{k}$.

ПРЕДЛОЖЕНИЕ 6.1.1. (i) Любой наклонный модуль из $\widetilde{\mathscr{O}}_{k}$ есть прямая сумма неразложимых наклонных модулей. Для любого $(\mu, k)$, где $\mu$ - челочисленный доминантный вес, имеется единственный неразложимьй наклонный 
модуль $\widetilde{U}_{\mu, k}$, такой, что

$$
\operatorname{ch}\left(\widetilde{U}_{\mu, k}\right)=\operatorname{ch}\left(V_{\mu}^{k}\right)+\sum_{\nu<_{k} \mu} c_{\nu} \operatorname{ch}\left(V_{\nu}^{k}\right) .
$$

( Частичный порядок $<_{k}$ определяется стандартным образом с помощью порядка Брюа на аффинной аруппе Вейля.) Отображение $\mu \mapsto \widetilde{U}_{\mu, k}$ устанавливает биекиию между множеством весов, удовлетворяюших упомянутому выше условию, и множеством классов изоморфизма неразложимых наклонньлх модулей в $\widetilde{\mathscr{O}}_{k}$.

(ii) Сходным образом, любой наклонный модуль из $\mathscr{O}_{k}$ есть прямая сумма неразложимых наклонных модулей. Для любого $(\mu, k)$ существует единственный неразложимый наклонный модуль $U_{\mu, k}$, такой, что

$$
\operatorname{ch}\left(U_{\mu, k}\right)=\operatorname{ch}\left(M_{\mu}^{k}\right)+\sum_{\nu<_{k} \mu} c_{\nu} \operatorname{ch}\left(M_{\nu}^{k}\right) .
$$

Отображение $(\mu, k) \mapsto U_{\mu, k}$ устанавливает биекиию между множеством весов и множеством классов изоморфизма неразложимьх наклонньх модулей u3 $\mathscr{O}_{k}$.

ДокАЗАтЕЛьство. Утверждение (i) доказано в [23] с использованием общих результатов Рингеля [26]; (ii) может быть доказано таким же образом. Прозрачное изложение, приспособленное для категории $\mathscr{O}$, имеется в [2].

СлЕдСТВИЕ 6.1.2. Множество характеров наклонных модулей из $\mathscr{O}_{k}\left(\widetilde{\mathscr{O}}_{k}\right)$ - базис кольиа Гротендика на $\mathscr{O}_{k}\left(\widetilde{\mathscr{O}}_{k}\right)$.

Каждан и Люстиг доказали, что категория $\widetilde{\mathscr{O}}_{k}$ жесткая. Некоторые следствия этого факта собраны в следующей лемме.

Лемма 6.1.3. (i) Для любого $A \in \widetilde{\mathscr{O}}_{k}$ Функтор $A \dot{\otimes} ?: \mathscr{O}_{k} \rightarrow \mathscr{O}_{k}$ точен.

(ii) Для любых $A \in \widetilde{\mathscr{O}}_{k}, B \in \mathscr{O}_{k}$ виполняется равенство $D(A \dot{\otimes} B)=$ $D(A) \dot{\otimes} D(B)$.

(iii) Если оба модуля $A \in \widetilde{\mathscr{O}}_{k}, B \in \mathscr{O}_{k}$ наклонные, то таков же и $A \dot{\otimes} B$.

ДокАЗАтЕльство. (i) доказывается так же, как следствие 4.2.4.

(ii) - общий факт о моноидальных категориях, см. [23, III, Proposition A. 1].

(iii) — немедленное следствие п. (ii) и того факта, что $\dot{\otimes}$-произведение модуля с фильтрацией Вейля и модуля с фильтрацией Верма есть модуль с фильтрацией Верма; см замечание 4.4.2.

\section{2. Наклонные функторы.}

6.2.1. Модули, зависящие от параметра. Пусть $t$ - переменная, а $R(t)-$ кольцо рациональных функций с полюсами только на положительной части действительной оси. Наряду с $\mathscr{O}_{k}$ введем категорию $\mathscr{O}_{t}$, определенную так же, как $\mathscr{O}_{k}$, за исключением того, что $\mathbb{C}$ заменено на $R(t)$, т. е. $\hat{\mathfrak{g}}$ рассматривается как $R(t)$-алгебра, а от объектов категории $\mathscr{O}_{t}$ требуется, чтобы они были свободными $R(t)$-модулями. Возникает функтор специализации

$$
s p_{k}: \mathscr{O}_{t} \rightarrow \mathscr{O}_{k}, \quad M \mapsto M(k) \stackrel{\text { def }}{=} M /(t-k) M .
$$

Пусть $\mathscr{A}_{t} \subset \mathscr{O}_{t}$ — полная подкатегория, состоящая из таких $V$, что $V(k)$

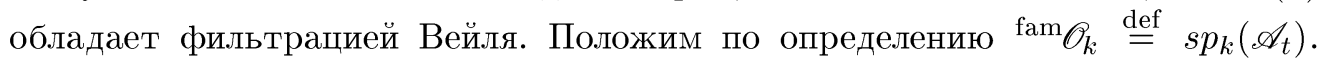




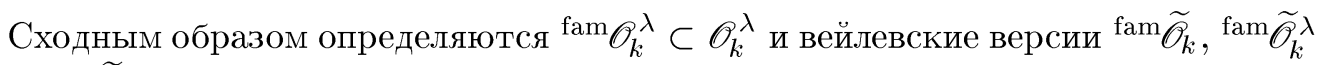
$\subset \operatorname{fam} \widetilde{\mathscr{O}}_{k}$.

Примеры. (i) $V_{\lambda}^{k} \in$ fam $\widetilde{\mathscr{O}}_{k}, M_{\lambda}^{k} \in$ fam $\mathscr{O}_{k}$.

(ii) $V_{\lambda}^{k} \dot{\otimes} V_{\mu}^{k} \in{ }^{\text {fam } \widetilde{O}_{k}}, V_{\mu}^{k} \dot{\otimes} M_{\lambda}^{k} \in{ }^{\text {fam } \mathscr{O}_{k}}$.

(iii) Прямые слагаемые модулей из п. (ii) принадлежат соответствующим подкатегориям.

6.2.2. Основные результаmы. Начиная с этого момента, у нас будут зафиксированы два антидоминантных веса $\left(\lambda_{r}, k\right)$ и $\left(\lambda_{l}, k\right)$, такие, что $\lambda_{r}, \lambda_{l}$ целочисленны.

ОПРЕДЕЛЕНИЕ. Наклонныл функтором называется прямое слагаемое функтора

$$
p_{\lambda_{l}} \circ(U \dot{\otimes} ?):{ }^{\text {fam }} \mathscr{O}_{k}^{\lambda_{r}} \rightarrow{ }^{\text {fam }} \mathscr{O}_{k}^{\lambda_{l}}
$$

где $U$ - наклонный модуль.

Вот один из наших главных результатов.

ТЕОРЕма 6.2.1. (i) Каждый наклонный функтор является прямой суммой неразложимых.

(ii) Имеется биекция между наклонными функторами и элементами групnы $W_{k}$. Функтор $\Phi_{w} \in \operatorname{Funct}\left({ }^{\text {fam }} \mathscr{O}_{k}^{\lambda_{r}},{ }^{\text {fam } \mathscr{O}_{k}^{\lambda_{l}}}\right)$, coответствуюиий $w \in W_{k}$, однозначно определяется условием $\Phi_{w}\left(M_{\lambda_{r}}^{k}\right)=U_{w \cdot \lambda_{l}, k} \cdot$

Одним из следствий этой теоремы является то, что категория наклонных

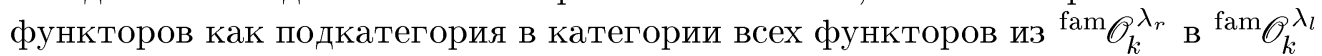
полупроста и эквивалентна категории наклонных модулей в $\mathscr{O}_{k}^{\lambda_{l}}$. Обозначим эту категорию через $\operatorname{Tilt}\left(\lambda_{l}, \lambda_{r}\right)$.

Предположим теперь, что у нас есть три антидоминантных веса $\left(\lambda_{1}, k\right)$, $\left(\lambda_{2}, k\right),\left(\lambda_{3}, k\right)$ и два наклонных функтора $\Phi \in \operatorname{Tilt}\left(\lambda_{2}, \lambda_{1}\right)$ и $\Psi \in \operatorname{Tilt}\left(\lambda_{3}, \lambda_{2}\right)$. Тогда $\Psi \circ \Phi$ - тоже наклонный функтор, что вытекает из леммы 6.1.3(iii) и наличия морфизма ассоциативности $U_{2} \dot{\otimes}\left(U_{1} \dot{\otimes} B\right)=\left(U_{2} \dot{\otimes} U_{1}\right) \dot{\otimes} B$. Если к тому же $\lambda_{l}=\lambda_{r}=\lambda$, то категория $\operatorname{Tilt}(\lambda) \stackrel{\text { def }}{=} \operatorname{Tilt}\left(\lambda_{r}, \lambda_{l}\right)$ замкнута относительно ○ и пара (Tilt $(\lambda)$, 。) является полупростой моноидальной категорией. Следующая теорема - наш второй главный результат, касающийся наклонных функторов.

ТЕорема 6.2.2. Кольцо Гротендика категории (Tilt $(\lambda)$, 。) изоморфно групповой алгебре группь $W_{k}$.

Доказательства двух последних теорем будут опубликованы позже.

\section{ЛитеРАтУРА}

1. Adamovic D., Milas A. Vertex operator algebras associated to modular invariant representations for $A_{1}^{1}$. Math. Res. Lett., 2, No. 3, 563-575 (1995).

2. Andersen H., Paradowski J. Fusion categories arising from semi-simple Lie algebras. Comm. Math. Phys., 169, 563-588 (1995).

3. Bernstein J. N., Gelfand S. I. Tensor products of finite and infinite dimensional representations of semi-simple Lie algebras. Compositio Math., 41, No. 2, 245-285 (1980).

4. Deodhar V. V., Gabber O., Kac V. G. Structure of some categories of representations of infinite dimensional Lie algebras. Adv. Math., 45, 92-116 (1982). 
5. Дринфельд В. Г. О квазитреугольных квазихопфовых алгебрах и одной группе, тесно связанной с $\operatorname{Gal}(\bar{Q} / Q)$. Алгебра и анализ, 2, вып. 4, 149-181 (1990).

6. Dong $\mathrm{C}$., Li H., Mason G. Vertex operator algebras associated to admissible representations of $\widehat{s l}_{2}$. Preprint q-algebra/9509026.

7. Duflo $M$. Representations irreductibles des groupes semi-simples complexes. In: Lect. Notes in Math., Vol. 497, 1975, pp. 26-88.

8. Enright T. On the irreducibility of the fundamental series of a real semi-simple Lie algebra. Ann. Math., 110, 1-82 (1979).

9. Feigin B., Frenkel E. Affine Kac-Moody algebras at the critical level and GelfandDikii algebras. Int. J. Math. Phys. A, 7, Supplement 1A, 197-215 (1992).

10. Feigin B., Malikov F. Fusion algebras and cohomology of a nilpotent subalgebra of an affine Lie algebra. Lett. Math. Phys., 31, 315-325 (1994).

11. Feigin B., Malikov F. Modular functor and representation theory of $\widehat{s l}_{2}$ at a rational level. In: "Operads: Proceedings of Renaissance Conferences", Contemp. Math., vol. 202 (J-L. Loday, J. Stasheff, and A. Voronov, eds.), 1997.

12. Finkelberg M. Fusion Categories, Ph.D. Thesis, Harvard University, 1993.

13. Frenkel E., Kac V., Wakimoto $M$. Characters and fusion rules for W-algebras via quantized Drinfeld-Sokolov reduction. Commun. Math. Phys., 147, 295-328 (1992).

14. Frenkel I. B., Lepowsky J., Meurman A. Vertex operator algebras and the monster, Academic Press, 1988.

15. Frenkel I. B., Zhu Y. Vertex operator algebras associated to representations of affine and Virasoro algebras. Duke Math. J., 66, 123-168 (1992).

16. Jantzen J. C. Moduln mit einem hoschten Gewicht. Lect. Notes in Math., vol. 750, Springer-Verlag, 1979.

17. Jantzen J. C. Representations of Algebraic Groups. Pure Appl. Math., Vol. 131, Academic Press, 1987.

18. Joseph A. Dixmier's problem for Verma and principal series submodules. J. London Math. Soc. (2), 20, No. 2, 193-204 (1979).

19. Kac V. G., Kazhdan D. A. Structure of representations with highest weight of infinite dimensional Lie algebras. Adv. Math., 34, 97-108 (1979).

20. Kac V. G., Wakimoto $M$. Modular invariant representations of infinite dimensional Lie algebras and superalgebras. Proc. Nat. Acad. Sci. U.S.A., 85, No. 14, 4956-4960 (1988).

21. Kazhdan D., Lusztig G. Affine Lie algebras and quantum groups. Internat. Math. Res. Notices, No. 2, 21-29 (1991).

22. Kazhdan D., Lusztig G. Tensor structures arising from affine algebras. I, II. J. Amer. Math. Soc., 6, No. 4, 905-947, 949-1011 (1993).

23. Kazhdan D., Lusztig G. Tensor structures arising from affine algebras. III, IV. J. Amer. Math. Soc., 7, No. 2, 335-381, 383-453 (1994).

24. Lusztig $G$. Nonlocal finiteness of a $W$-graph. Electronic Journal of Representation Theory, 1, 25-30 (1997).

25. Mircovič $I$. unpublished.

26. Ringel $C$. $M$. The category of modules with good filtrations over a quasi-hereditary algebra has almost split sequences. Math. Z., 208, 209-223 (1991).

27. Rocha A., Wallach N. Projective modules over graded Lie algebras I. Math. Z., 180, 151-177 (1982).

28. Vogan D. Irreducible characters of semi-simple Lie groups. Duke Math. J., 46, 61-108, 805-859 (1979).

29. Zuckerman $G$. Tensor products of finite and infinite dimensional representations of semi-simple Lie groups. Ann. of Math., 106, 295-308 (1977). 